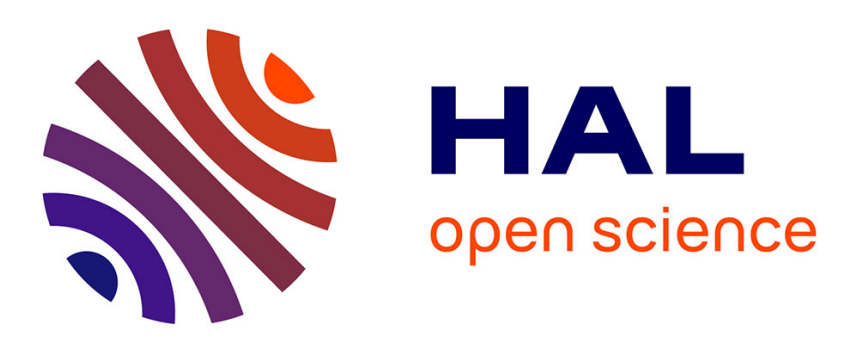

\title{
Numerical and Experimental Study of the Hydrodynamic Phenomena in Heterogeneous Sea Surface EM Bistatic Scattering.
}

Slah-Eddine Ben Khadra, Ali Khenchaf

\section{- To cite this version:}

Slah-Eddine Ben Khadra, Ali Khenchaf. Numerical and Experimental Study of the Hydrodynamic Phenomena in Heterogeneous Sea Surface EM Bistatic Scattering.. OCEANS'11, Jun 2011, Santander, Spain. hal-00636413

HAL Id: hal-00636413

https://hal-ensta-bretagne.archives-ouvertes.fr/hal-00636413

Submitted on 27 Oct 2011

HAL is a multi-disciplinary open access archive for the deposit and dissemination of scientific research documents, whether they are published or not. The documents may come from teaching and research institutions in France or abroad, or from public or private research centers.
L'archive ouverte pluridisciplinaire HAL, est destinée au dépôt et à la diffusion de documents scientifiques de niveau recherche, publiés ou non, émanant des établissements d'enseignement et de recherche français ou étrangers, des laboratoires publics ou privés. 


\title{
Numerical and Experimental Study of the Hydrodynamic Phenomena in Heterogeneous Sea Surface EM Bistatic Scattering.
}

\author{
S. Ben Khadra, A. Khenchaf \\ Ensta Bretagne, E3I2-EA3876(REMS) \\ 2, rue François Verny 29806 Brest Cedex 09, France \\ (benkhasl, Ali.Khenchaf)@ensta-bretagne.fr
}

\begin{abstract}
:
In this paper, we represent numerical results which, validate our former work on the hydrodynamic effect (nonlinear) produced by breaking waves [1] in EM bistatic scattering coefficients.

We calculated the scattering coefficient $\sigma_{H H}$ of breaking waves in bi-static configurations as a function of time. Then, in this paper we represent the first experimental measurements, which have been carried out in X-band in our anechoic chamber (E $E^{3} I^{2}-E A 3876-$ laboratory of Ensta bretagne); of the breaking waves profiles. In this work, we consider the sea surface as a perfect conductor rough surface.
\end{abstract}

\section{INTRODUCTION}

Many physical phenomena coexist and affect the electromagnetic wave propagation over a heterogeneous sea surface (due to the refractive index gradients, roughness of the sea surface, the presence of objects, pollutants, ship wake, areas Coastal, ...). In recent literature, the study of these former phenomena is generally done separately. There is no real research that examined the interaction between these phenomena and their contribution to the EM surface scattering containing breaking waves. The work presented in this paper focuses on the influence of nonlinear waves (breaking waves) on the EM signature of a sea surface

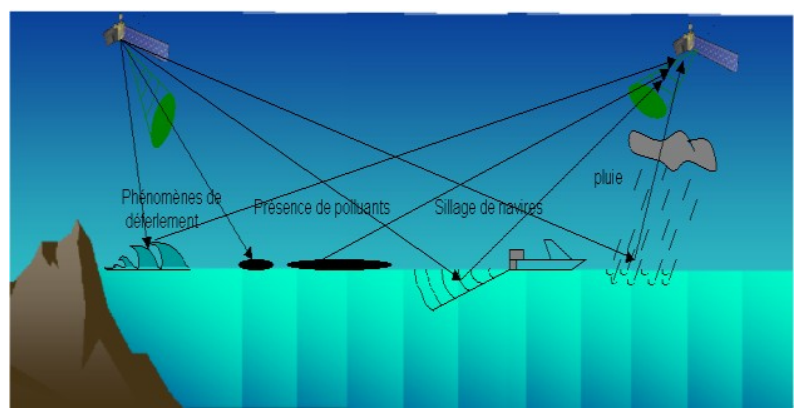

Fig. 1.The heterogeneous sea surface

In general, the scattering of an electromagnetic wave by the sea surface can be divided into two distinct phenomena. Initially, there is the scattering of linear waves and secondly, the scattering of breaking waves which cause non-linear phenomena. In our case, we could consider that the ocean surface is geometrical heterogeneous. These phenomena add a component which is not negligible and which is called non-
Bragg diffusion in the total diffusion coefficients. Therefore, we suggest writing the total component as the sum of two contributions; it is given by Eq. (1).

$$
\sigma=\sigma_{\text {Bragg }}+\sigma_{\text {Mon }- \text { Bragg }}
$$

To date, the Bragg scattering (scattering of linear waves) has been well studied in many papers [2] [3] [4]. Other works and measures on rough surfaces observed in bistatic configuration were performed on stamp of soil with different textures, including allowing the estimation of parameters of natural surfaces from measurements made in an anechoic chamber [5]. However, the surface scattering by breaking waves "nonBragg" is not enough studied and especially in the bistatic configuration.

In our first results [1], we showed that for bistatic onfiguration (forward propagation) we can find the nonlinear effect that occurs as a peak of sea.

This peak can lead to false echo, which can be identified as virtual targets that later disorders the bi-static radar detection (false alarm). So to improve the detection and to reduce false alarm rates it is important to distinguish between targets and the peak of sea waves generated by breaking waves. For this we must study the electromagnetic signature of breaking waves, so that we can easily identify them.

In this paper we present the numerical results in the bistatic configuration where we can find some false echoes. Then, we represent the experimental radar measurement validations considering targets in the form of breaking waves.

\section{BREAKING WAVES}

The breaking is a dispersion process of the energy which corresponds with the last stage of a wave's life. During this phase, the wave is subject to important transformations in its behavior and structure. When the waves approach a shore, they arrive in waters less than half the depth of their wavelength. Their time period does not change, but their wavelength decreases, as does their speed, however their height increases. When the depth of the coastal water decreases, there is less free space for the water particles situated on the crest of the wave to move around in, therefore the wave breaks.

Recently, it has become possible to numerically simulate these types of waves. Here follows a presentation of the results of 
one of these simulations which is called LONGTANK [6] (fig. 2 ), and which will be used throughout this article as a breaking wave model.

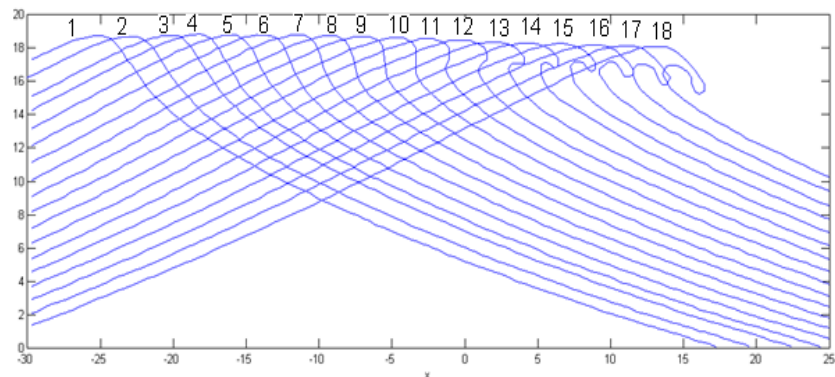

Fig. 2. Profile of breaking waves [6]

LONGTANK is a hydrodynamic code developed at the University of California, Santa Barbara (UCSB), they have devised a numerical wave basin, for the study of groups of waves, the wave-wave interactions, the deforming of waves, the breaking of waves, and other non-linear effects. Their calculations of wave forms are coherent with experimental measures and observations of the ocean. The 18 waves used in this investigation belong to Case 2.4 (studied by Holliday) [7].

The sequence represents the temporal evolution of a breaking wave with a wavelength of $2.3 \mathrm{~m}$, and during a period of 1.212 seconds. Usually, there exist four types of diffusion mechanism of a breaking wave [8]. A single mechanism with a single trajectory is found and is the diffusion of the crest of the wave, and three mechanisms with multiple (double) trajectories, which are due to the propagation of the electromagnetic waves between the crest and the front side of the waves.

In this context, only the case of the single trajectory is examined since in the profile of the waves the front side of the waves has been eliminated, as shown by figure 3 .

\section{METHOD OF CALCULATION}

The numerical FBM is used to calculate the scattering coefficient.

The FBM has been well-discussed in [9] [10]. The integral equation of the electronic field (EFIE) for a perfect conductor (equation 2) is discrete under a matrix form (equation 3 ).

$$
\overline{\mathrm{E}}(\overline{\mathrm{r}})=\overline{\mathrm{E}}_{\text {inc }}(\overline{\mathrm{r}})+\mathrm{i} \cdot \omega \cdot \mu_{0} \int \overline{\overline{\mathrm{G}}}\left(\overline{\mathrm{r}}, \overline{\mathrm{r}}^{\prime}\right) \cdot \overline{\mathrm{J}}\left(\overline{\mathrm{r}}^{\prime}\right) \mathrm{d} \mathrm{S}^{\prime}
$$

where $J(r)$ is the induced current on the surface, $\omega$ is The radian frequency, $\mu_{0}$ is permeability of free space, $G$ is the dyadic Green's function for free space and Einc is the incident wave.

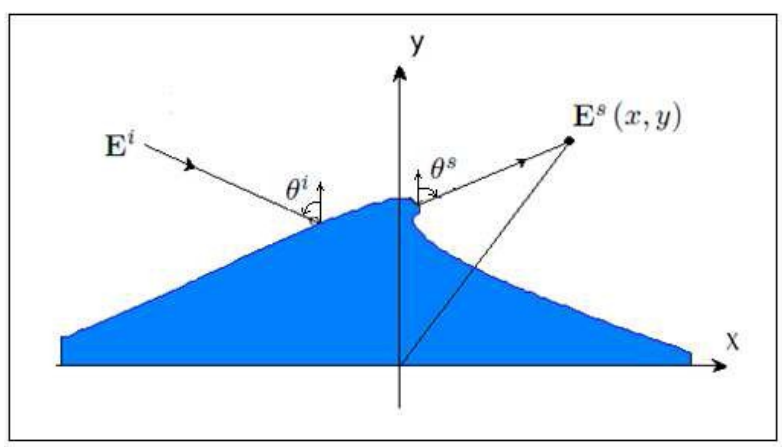

Figure.3. The geometry of diffusion for a breaking wave

$$
\mathrm{Z} . \mathrm{I}=\mathrm{V}
$$

Where $\mathrm{Z}$ it is the impedance of the matrix, $\mathrm{V}$ is the wave of incidence, and I is the induced current along the length of the rough surface. The FBM method consists of breaking down the equation (3):

$$
\begin{gathered}
I=I f+I b \\
Z=Z_{f}+Z_{s}+Z b
\end{gathered}
$$

Where If is the forward component (the current contribution due to the waves propagating forwards), Ib is the backward component (current contribution due to the waves propagating backwards), and $\mathrm{Zf}, \mathrm{Zs}$ and $\mathrm{Zb}$ are respectively, the lower triangular part, the diagonal part, and the upper triangular part of Z .

After the breakdown, the resolution of the equation (3) (determined by I) becomes:

$$
\begin{aligned}
& \left(Z_{s}+Z_{f}\right) \cdot I f=V-Z_{f} \cdot I b \\
& \left(Z_{s}+Z b\right) \cdot I b=-Z b \cdot I f
\end{aligned}
$$

This system can resolve itself by iteration:

$$
\begin{gathered}
\left(Z_{s}+Z_{f}\right) \cdot \operatorname{If}(i)=V-Z_{f} \cdot I b \cdot(i-1) \\
(Z s+Z b) \cdot \operatorname{Ib}(i)=-Z_{b} \cdot I f(i)
\end{gathered}
$$

The iteration begins by $\operatorname{Ib}(0)=0$

The algorithm of FBM used in this section is in two dimensions $(\mathrm{x}, \mathrm{y})$. An infinite extension is created in the third dimension $(z)$, in which none of the properties vary.

\section{NUMERICAL RESULTS}

To analyse the hydrodynamic effect of breaking waves (sea spike) we represented the evolution $\sigma_{\mathrm{HH}}$ for the profiles 4,8 , 12 and 16, (Figure 4). 


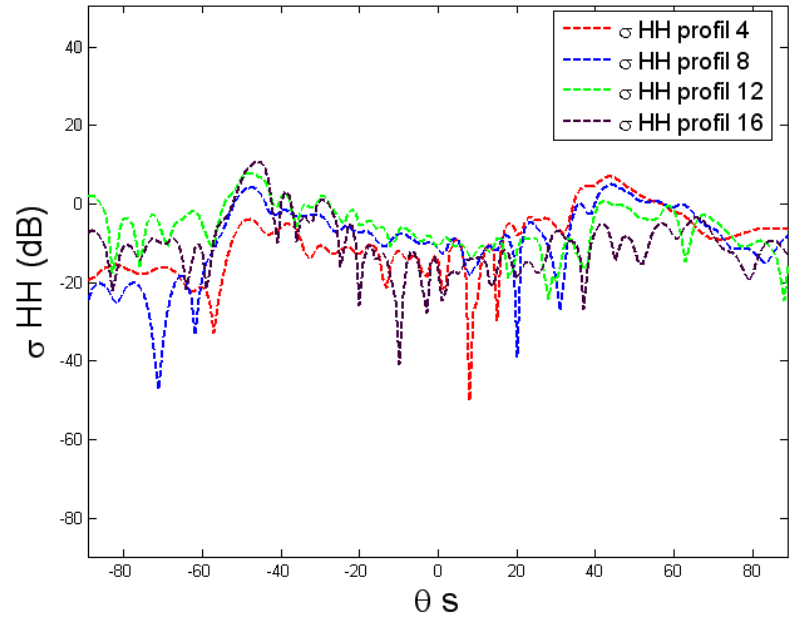

Fig.4. Bistatic scattering coefficient $\sigma_{\mathrm{HH}}$ of profiles $4,8,12$, and $16\left(\theta \mathrm{i}=0^{\circ}\right)$

Wetzel, [11] has defined the sea spike as a rapid changing of $\sigma_{\mathrm{HH}}$ exceeding $10 \mathrm{~dB}$ in a period of about $100 \mathrm{~ms}$.

For the bi-static configuration $\left(\theta \mathrm{i}=0^{\circ}\right.$ and $\theta \mathrm{s}$ between $-42^{\circ}$ and $-50^{\circ}$ ) we find out an increase of $\sigma_{\mathrm{HH}}$, which is the response of the positive slope of the wave. To understand better this augmentation, we plot evolution of $\sigma_{\mathbf{H H}}$ as a function of time. Indeed, we calculate $\sigma_{\mathrm{HH}}$ for the 16 breaking waves profiles (Fig. 5), for the bi-static configuration as follows $\theta \mathrm{i}=0^{\circ}, \theta \mathrm{s}=-$ $42^{\circ}, \theta \mathrm{i}=0^{\circ}, \theta \mathrm{s}=-45^{\circ}$ and $\left.\theta \mathrm{i}=0^{\circ}, \theta \mathrm{s}=-50^{\circ}\right)$ :

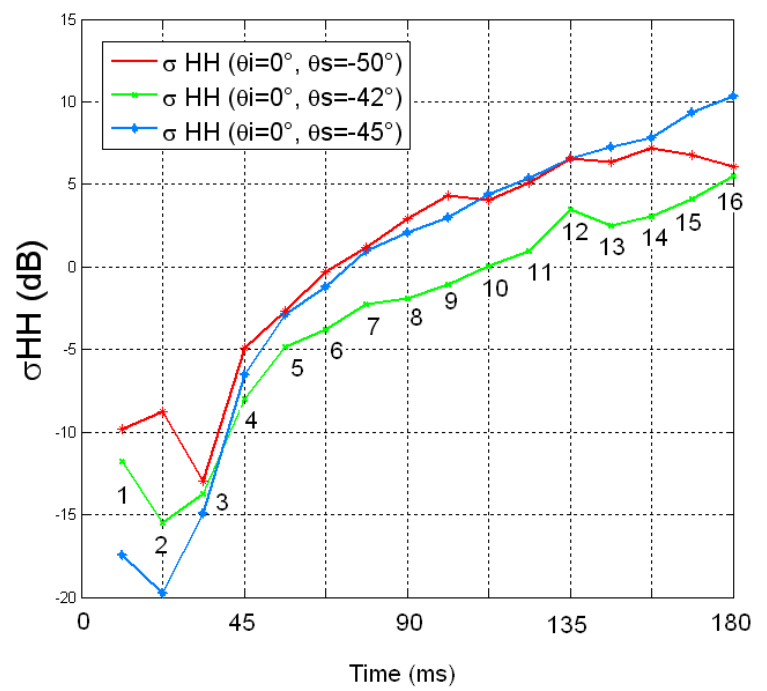

Fig.5.Bistatic scattering coefficients $\sigma_{\mathrm{HH}}$ of profile 16

The table below shows the difference of $\sigma_{\mathrm{HH}}(\mathrm{dB})$ between the profile 12 and profile 3 .

\begin{tabular}{|l|c|}
\hline$\theta \mathrm{i}=0^{\circ}$ & $\sigma_{\mathrm{HH}}(\mathrm{dB})$ of profile $12-\sigma_{\mathrm{HH}}(\mathrm{dB})$ of profile 3 \\
\hline$\theta \mathrm{s}=-42^{\circ}$ & $+17.19 \mathrm{~dB}$ \\
\hline$\theta \mathrm{s}=-45^{\circ}$ & $+21.53 \mathrm{~dB}$ \\
\hline$\theta \mathrm{s}=-50^{\circ}$ & $+19.56 \mathrm{~dB}$ \\
\hline
\end{tabular}

Knowing that the time between profile 3 and profile 12 is in order of $100 \mathrm{~ms}$ and the difference of the value of $\sigma_{\mathrm{HH}}(\mathrm{dB})$ between these two profile exceeds $10 \mathrm{~dB}$, which satisfies the criterion of Wetzel [11] for a sea spike. Then, we can say that for the bi-static configuration $\left(\theta \mathrm{i}=0^{\circ}, \theta \mathrm{s}=-42^{\circ}, \theta \mathrm{i}=0^{\circ}, \theta \mathrm{s}=-45^{\circ}\right.$ and $\theta \mathrm{i}=0^{\circ}, \theta \mathrm{s}=-50^{\circ}$ ) we find out the nonlinear effect of a breaking wave which is in the form of a sea peak.

In this way we confirm our initial results [1]. Indeed it is the scattering of the positive slope of the breaking wave, which causes the sea spike.

\section{VALIDATION BY EXPERIMENTAL RADAR MESUREMENTS}

We represent in this part the first radar measurements performed in our anechoic chamber to validate our numerical results given in [1].

We fristly show the measurements of profile 12 of the breaking wave, using a metalic profile (Figure 6).

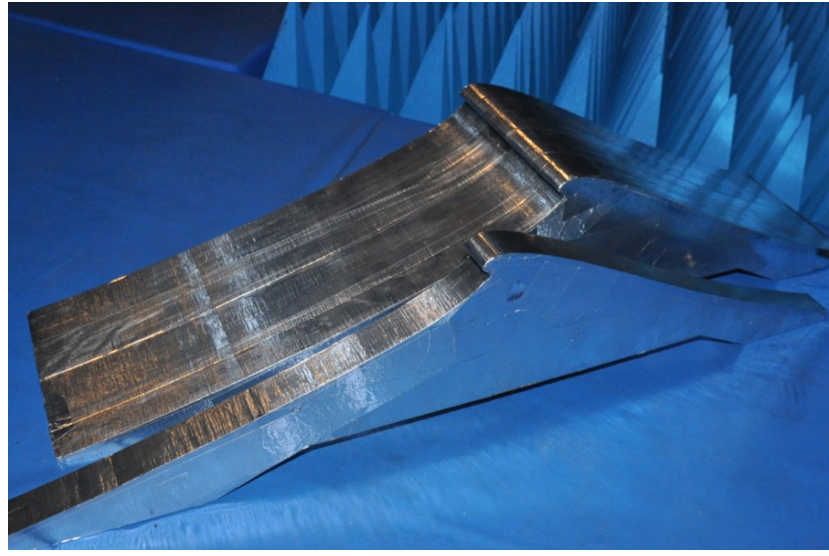

Fig.6. the metalic profile 12 of the breaking wave

If we consider that geometry of the profile is constant along the axis $(Z)$, it is enough to take a metalic profile with small thickness $(3 \mathrm{~cm})$. And thus we can compare our experimental measurements with 1D numerical simulation.

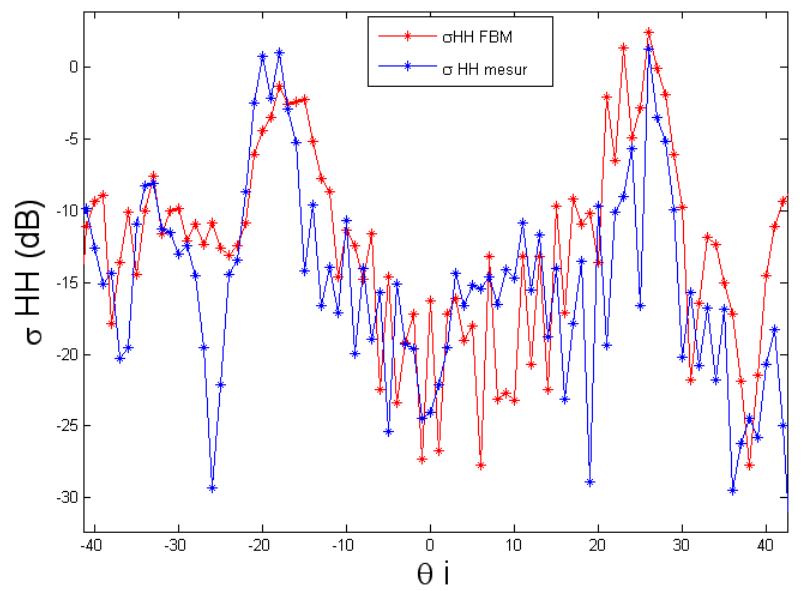

Fig.7.monostatic scattering coefficients $\sigma_{\mathrm{HH}}(\mathrm{FBM}$ and measurements) 
These first experimental measurements are in perfect agreement with our numerical simulations (Figure 7). Thus, these first results are encouraging to make measurements for other configuration.

\section{CONCLUSION :}

In this paper we validated our earlier work [1]. Indeed, for the bistatic configuration (forward propagation) and for nongrazing angles we found out the nonlinear effect, which is in the form of a pike sea, while the hydrodynamic effect is only for grazing angles for monostatic configuration (Holliday.[7]).

These results demonstrate the importance of the breaking waves in the calculation of the electromagnetic signature of heterogeneous marine coastal areas in bistatic configuration.

We also represented our first radar measurements of monostatic configuration validation conducted with metallic which has breaking waves. In our future work we will continue to carry out experimental radar measurements for different profiles of breaking waves and for the bistatic configuration to validate our numerical results.

\section{REFERENCES}

[1] S. Ben Khadra, A. Khenchaf "The bistatic electromagnetic signature of heterogeneous sea surface: Study of the hydrodynamic phenomena" IGARSS 2010.

[2] A.Khenchaf, "Bistatic scatering and depolarization by randomly rough surface : application to the natural rough surfaces in x-band," 2000.

[3] A.Awada, Y. Ayari, A.Khenchaf, and A.Coatanhay, "Bistatic scattering from an anisotropic sea surface: Numerical comparison between the first-order ssa and the tsm models," Waves in Random and Complex Media, vol. 16(3), 2006.

[4] N.Sajjad, A.Khenchaf, A.Coatanhay, and A.Awada, "An improved two-scale model for the ocean surface bistatic scattering," IGARSS, Boston, USA, pp. 6-11, 2008

[5] Khadra, K. B., ISurface parameter estimation using bistatic polarimetric X-band measurements," Ph.D. thesis, German

Aerospace Centre (DLR), Institute of Radio Frequency Technol-ogy, Oberpfa®enhofen, Germany, 2008.

[6]Wang P, Yao, and Tulin, "An efficient numerical tank for non-linear water waves, based on the multi-subdomain approach with bem," INumerical Methods in Fluids, vol. 20, pp. 1315-1336, 1995.
[7] D. Holliday, L. L. DeRaad Jr, and G. J. St-Cyr, "Seaspike backscatter from a steepening wave," IEEE Trans. Antennas Propagation, vol. 46, pp. 108113, 1998.

[8] J.T.Johnson, "Radar image study of simulated breaking waves," 2002.

[9] D. Holliday, L.L. DeRaad, and G.J. St-Cyr, "Forwardbackward method for scattering from imperfect conductors," EEE Transactions on Antennas and Propagation.

[10] H.T. Chan and J.T. Johnson, "Formulation of forwardbackward method using novel spectral acceleration for the modeling of scattering from impedance rough surfaces," IEEE Transactions on Geosicence and Remote Sensing, vol. 38(1), pp. 605-607.vol. 46(1).

[11] L. B.Wetzel and M. I. Skolnik. J., "Sea clutter," Ed. New York: McGraw-Hill, 1990. 